\title{
Somewhat fuzzy irresolute continuous mappings
}

\author{
Young Bin $\mathrm{Im}^{1, *}$, Joo Sung $\mathrm{Lee}^{2}$ and Yung Duk Cho ${ }^{1}$ \\ 1 Faculty of General Education, Dongguk University, Seoul 100-715, Korea \\ 2 Department of Mathematics, Dongguk University, Seoul 100-715, Korea
}

\begin{abstract}
We define and characterize a somewhat fuzzy irresolute continuous mapping and a somewhat fuzzy irresolute semiopen mapping on a fuzzy topological space. Besides, some interesting properties of those mappings are given.
\end{abstract}

Key Words: somewhat fuzzy irresolute continuous mapping, somewhat fuzzy irresolute semiopen mapping.

\section{Introduction}

The class of somewhat continuous mappings was first introduced by Gentry and others [2]. Later, the concept of "somewhat" in classical topology has been extended to fuzzy topological spaces. In fact, somewhat fuzzy continuous mappings and somewhat fuzzy semicontinuous mappings on fuzzy topological spaces were introduced and studied by G. Thangaraj and G. Balasubramanian in [5] and [6] respectively.

Meanwhile, the concept of fuzzy irresolute continuous mapping on fuzzy topological space was introduced and studied by M. N. Mukherjee and S. P. Shina in [3].

In this paper, the concepts of somewhat fuzzy irresolute continuous mappings and somewhat fuzzy irresolute semiopen mappings on a fuzzy topological space are introduced and we characterize those mappings. Besides, some interesting properties of those mappings are also given.

\section{Preliminaries}

Throughout this paper, we denote $\mu^{c}$ with the complement of the fuzzy set $\mu$ on a nonempty set $X$, which is defined by $\mu^{c}(x)=(1-\mu)(x)=1-\mu(x)$ for all $x \in X$. If $\mu$ is a fuzzy set on a nonempty set $X$ and if $\nu$ is a fuzzy set on a nonempty set $Y$, then $\mu \times \nu$ is a fuzzy set on $X \times Y$, defined by $(\mu \times \nu)(x, y)=\min (\mu(x), \nu(y))$ for every $(x, y) \in X \times Y$. Let $f: X \rightarrow Y$ be a mapping and let $\mu$ be a fuzzy set on $X$. Then $f(\mu)$ is a fuzzy set on $Y$

Manuscript received Oct. 13, 2011; revised Oct. 4, 2012; accepted Oct 4, 2012.

*Corresponding Author: Young Bin Im (philpen@dongguk.edu)

(c)The Korean Institute of Intelligent Systems. All rights reserved. defined by

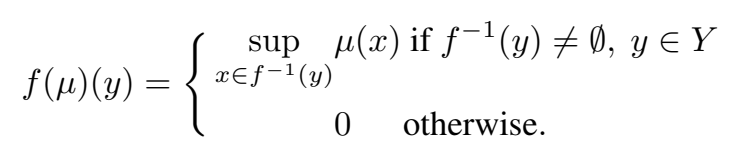

Let $\nu$ be a fuzzy set on $Y$. Then $f^{-1}(\nu)$ is a fuzzy set on $X$, defined by $f^{-1}(\nu)(x)=\nu(f(x))$ for each $x \in X$. The graph $g: X \rightarrow X \times Y$ of $f$ is defined by $g(x)=$ $(x, f(x))$ for each $x \in X$. Then $g^{-1}(\mu \times \nu)=\mu \wedge f^{-1}(\nu)$. The product $f_{1} \times f_{2}: X_{1} \times X_{2} \rightarrow Y_{1} \times Y_{2}$ of mappings $f_{1}: X_{1} \rightarrow Y_{1}$ and $f_{2}: X_{2} \rightarrow Y_{2}$ is defined by $\left(f_{1} \times\right.$ $\left.f_{2}\right)\left(x_{1}, x_{2}\right)=\left(f_{1}\left(x_{1}\right), f_{2}\left(x_{2}\right)\right)$ for each $\left(x_{1}, x_{2}\right) \in X_{1} \times$ $X_{2}[1]$.

Now let $X$ and $Y$ be fuzzy topological spaces. We denote Int $\mu$ and $\mathrm{Cl} \mu$ with the interior and with the closure of the fuzzy set $\mu$ on a fuzzy topological space $X$ respectively. Then (i) $1-\mathrm{Cl} \mu=\operatorname{Int}(1-\mu)$ and (ii) $\mathrm{Cl}(1-\mu)=1-\operatorname{Int} \mu$.

We say that a fuzzy topological space $X$ is product related to a fuzzy topological space $Y$ if, for a fuzzy set $\mu$ on $X$ and $\nu$ on $Y, \gamma \geq \mu$ and $\delta \ngtr \nu$ (in which case $\left.\left(\gamma^{c} \times 1\right) \vee\left(1 \times \delta^{c}\right) \geq(\mu \times \nu)\right)$ where $\gamma$ is fuzzy open set on $X$ and $\delta$ is a fuzzy open set on $Y$, then there exists a fuzzy open set $\gamma_{1}$ on $X$ and a fuzzy open set $\delta_{1}$ on $Y$ such that $\gamma_{1}^{c} \geq \mu$ or $\delta_{1}^{c} \geq \nu$ and $\left(\gamma_{1}^{c} \times 1\right) \vee\left(1 \times \delta_{1}^{c}\right)=$ $\left(\gamma^{c} \times 1\right) \times\left(1 \times \delta^{c}\right)$.

A mapping $f: X \rightarrow Y$ is called fuzzy continuous if $f^{-1}(\nu)$ is a fuzzy open set on $X$ for any fuzzy open set $\nu$ on $Y$. And a mapping $f: X \rightarrow Y$ is called fuzzy open if $f(\mu)$ is a fuzzy open set on $Y$ for any fuzzy open set $\mu$ on $X$.

A fuzzy set $\mu$ on a fuzzy topological space $X$ is called fuzzy semiopen if $\mu \leq \mathrm{Cl} \operatorname{Int} \mu$ and $\mu$ is called fuzzy semiclosed if $\mu^{c}$ is a fuzzy semiopen set on $X$.

A mapping $f: X \rightarrow Y$ is called fuzzy semicontinuous if $f^{-1}(\nu)$ is a fuzzy semiopen set on $X$ for any fuzzy 
open set $\nu$ on $Y$. And a mapping $f: X \rightarrow Y$ is called fuzzy semiopen if $f(\mu)$ is a fuzzy semiopen set on $Y$ for any fuzzy open set $\mu$ on $X[1]$.

A mapping $f: X \rightarrow Y$ is called fuzzy irresolute continuous if $f^{-1}(\nu)$ is a fuzzy semiopen set on $X$ for any fuzzy semiopen set $\nu$ on $Y$ and a mapping $f: X \rightarrow Y$ is called fuzzy irresolute semiopen if $f(\mu)$ is a fuzzy semiopen set on $Y$ for any fuzzy semiopen set $\mu$ on $X$. It is clear that every fuzzy irresolute continuous mapping is a fuzzy semicontinuous mapping. And every fuzzy irresolute semiopen mapping is a fuzzy semiopen mapping from the above definitions. But the converses are not true in general [3].

A fuzzy set $\mu$ on a fuzzy topological space $X$ is called fuzzy dense if there exists no fuzzy closed set $\nu$ such that $\mu<\nu<1$.

A mapping $f: X \rightarrow Y$ is called somewhat fuzzy continuous if there exists a fuzzy open set $\mu \neq 0_{X}$ on $X$ such that $\mu \leq f^{-1}(\nu) \neq 0_{X}$ for any fuzzy open set $\nu$ on $Y$. It is clear that every fuzzy continuous mapping is a somewhat fuzzy continuous mapping. But the converse is not true in general.

A mapping $f: X \rightarrow Y$ is called somewhat fuzzy open if there exists a fuzzy open set $\nu \neq 0_{Y}$ on $Y$ such that $\nu \leq f(\mu) \neq 0_{Y}$ for any fuzzy open set $\mu$ on $X$. Note that every fuzzy open mapping is a somewhat fuzzy open mapping but the converse is not true in general [5]

A fuzzy set $\mu$ on a fuzzy topological space $X$ is called fuzzy semidense if there exists no fuzzy semiclosed set $\nu$ such that $\mu<\nu<1$.

A mapping $f: X \rightarrow Y$ is called somewhat fuzzy semicontinuous if there exists a fuzzy semiopen set $\mu \neq 0_{X}$ on $X$ such that $\mu \leq f^{-1}(\nu) \neq 0_{X}$ for any fuzzy open set $\nu$ on $Y$. It is clear that every fuzzy continuous mapping is a somewhat fuzzy semicontinuous mapping. But the converse is not true in general.

A mapping $f: X \rightarrow Y$ is called somewhat fuzzy semiopen if there exists a fuzzy semiopen set $\nu \neq 0_{Y}$ on $Y$ such that $\nu \leq f(\mu) \neq 0_{Y}$ for any fuzzy open set $\mu$ on $X$. Every fuzzy open mapping is a somewhat fuzzy semiopen mapping but the converse is not true in general [6].

\section{Somewhat fuzzy irresolute continuous mappings}

In this section, we introduce a somewhat fuzzy irresolute continuous mapping and a somewhat fuzzy irresolute semiopen mapping which are stronger than a somewhat fuzzy semicontinuous mapping and a somewhat fuzzy semiopen mapping respectively. And we characterize a somewhat fuzzy irresolute continuous mapping and a somewhat fuzzy irresolute open mapping.

Definition 3.1. A mapping $f: X \rightarrow Y$ is called somewhat fuzzy irresolute continuous if there exists a fuzzy semiopen set $\mu \neq 0_{X}$ on $X$ such that $\mu \leq f^{-1}(\nu) \neq 0_{X}$ for any fuzzy semiopen set $\nu \neq 0_{Y}$ on $Y$.

It is clear that every fuzzy irresolute continuous mapping is a somewhat fuzzy irresolute continuous mapping. And every somewhat fuzzy irresolute continuous mapping is a fuzzy semicontinuous mapping. Also, every fuzzy semicontinuous mapping is a somewhat fuzzy semicontinuous mapping from the above definitions. But the converses are not true in general as the following examples show.

Example 3.2. Let $\mu_{1}, \mu_{2}$ and $\mu_{3}$ be fuzzy sets on $X=$ $\{a, b, c\}$ and let $\nu_{1}, \nu_{2}$ and $\nu_{3}$ be fuzzy sets on $Y=$ $\{x, y, z\}$ with

$$
\begin{aligned}
& \mu_{1}(a)=0.1, \mu_{1}(b)=0.1, \mu_{1}(c)=0.1, \\
& \mu_{2}(a)=0.2, \mu_{2}(b)=0.2, \mu_{2}(c)=0.2, \\
& \mu_{3}(a)=0.5, \mu_{3}(b)=0.5, \mu_{3}(c)=0.5 \text { and } \\
& \nu_{1}(x)=0.3, \nu_{1}(y)=0.0, \nu_{1}(z)=0.3, \\
& \nu_{2}(x)=0.5, \nu_{2}(y)=0.5, \nu_{2}(z)=0.5, \\
& \nu_{3}(x)=0.5, \nu_{3}(y)=0.2, \nu_{3}(z)=0.5 .
\end{aligned}
$$

Let $\tau=\left\{0_{X}, \mu_{1}, \mu_{1}^{c}, 1_{X}\right\}$ be fuzzy topology on $X$ and let $\tau^{*}=\left\{0_{Y}, \nu_{1}, \nu_{2}, 1_{Y}\right\}$ be fuzzy topology on $Y$. Consider the mapping $f:(X, \tau) \rightarrow\left(Y, \tau^{*}\right)$ defined by $f(a)=y$, $f(b)=y$ and $f(c)=y$. Then we have $f^{-1}\left(\nu_{1}\right)=0_{X}$, $\mu_{1} \leq f^{-1}\left(\nu_{2}\right)=\mu_{3}$ and $\mu_{1} \leq f^{-1}\left(\nu_{3}\right)=\mu_{2}$. Since $\mu_{1}$ is a fuzzy semiopen set on $(X, \tau), f$ is somewhat fuzzy irresolute continuous. But $f^{-1}\left(\nu_{2}\right)=\mu_{3}$ and $f^{-1}\left(\nu_{3}\right)=$ $\mu_{2}$ are not fuzzy semiopen sets on $(X, \tau)$. Hence $f$ is not a fuzzy irresolute continuous mapping.

Example 3.3. Let $\mu_{1}, \mu_{2}$ and $\mu_{3}$ be fuzzy sets on $X=$ $\{a, b, c\}$ and let $\nu_{1}, \nu_{2}$ and $\nu_{3}$ be fuzzy sets on $Y=$ $\{x, y, z\}$ with

$$
\begin{aligned}
& \mu_{1}(a)=0.2, \mu_{1}(b)=0.2, \mu_{1}(c)=0.2, \\
& \mu_{2}(a)=0.3, \mu_{2}(b)=0.3, \mu_{2}(c)=0.3, \\
& \mu_{3}(a)=0.5, \mu_{3}(b)=0.5, \mu_{3}(c)=0.5 \text { and } \\
& \nu_{1}(x)=0.4, \nu_{1}(y)=0.0, \nu_{1}(z)=0.4, \\
& \nu_{2}(x)=0.5, \nu_{2}(y)=0.5, \nu_{2}(z)=0.5, \\
& \nu_{3}(x)=0.5, \nu_{3}(y)=0.2, \nu_{3}(z)=0.5 .
\end{aligned}
$$

Let $\tau=\left\{0_{X}, \mu_{2}, \mu_{1}^{c}, 1_{X}\right\}$ be fuzzy topology on $X$ and let $\tau^{*}=\left\{0_{Y}, \nu_{1}, \nu_{2}, 1_{Y}\right\}$ be fuzzy topology on $Y$. Consider 
the mapping $f:(X, \tau) \rightarrow\left(Y, \tau^{*}\right)$ defined by $f(a)=y$, $f(b)=y$ and $f(c)=y$. Since $f^{-1}\left(\nu_{1}\right)=0_{X}$ and $f^{-1}\left(\nu_{2}\right)=\mu_{3}$ are fuzzy semiopen sets on $(X, \tau), f$ is fuzzy semicontinuous. But the inverse image $f^{-1}\left(\nu_{3}\right)=$ $\mu_{1}$ of a fuzzy semiopen set $\nu_{3}$ on $\left(Y, \tau^{*}\right)$ is not fuzzy semiopen on $(X, \tau)$. Hence $f$ is not a fuzzy somewhat irresolute continuous mapping.

Example 3.4. Let $\mu_{1}, \mu_{2}$ and $\mu_{3}$ be fuzzy sets on $X=$ $\{a, b, c\}$ with

$$
\begin{aligned}
& \mu_{1}(a)=0.1, \mu_{1}(b)=0.1, \mu_{1}(c)=0.1, \\
& \mu_{2}(a)=0.3, \mu_{2}(b)=0.3, \mu_{2}(c)=0.3 \text { and } \\
& \mu_{3}(a)=0.8, \mu_{3}(b)=0.8, \mu_{3}(c)=0.8
\end{aligned}
$$

and let $\tau=\left\{0_{X}, \mu_{1}, \mu_{2}, 1_{X}\right\}$ and $\tau^{*}=\left\{0_{X}, \mu_{3}, 1_{X}\right\}$ be fuzzy topologies on $X$. Consider the identity mapping $i_{X}:(X, \tau) \rightarrow\left(X, \tau^{*}\right)$. We have $\mu_{2}^{c} \leq i_{X}^{-1}\left(\mu_{3}\right)=\mu_{3}$. Since $\mu_{2}^{c}$ is a fuzzy semiopen set on $(X, \tau), i_{X}$ is somewhat fuzzy semicontinuous. But $i_{X}^{-1}\left(\mu_{3}\right)=\mu_{3}$ is not a fuzzy semiopen set on $(X, \tau)$. Hence $i_{X}$ is not a fuzzy semicontinuous mapping.

Theorem 3.5. Let $f: X \rightarrow Y$ be a mapping. Then the following are equivalent:

(1) $f$ is somewhat fuzzy irresolute continuous.

(2) If $\nu$ is a fuzzy semiclosed set of $Y$ such that $f^{-1}(\nu) \neq 1_{X}$, then there exists a fuzzy semiclosed set $\mu \neq 1_{X}$ of $X$ such that $f^{-1}(\nu) \leq \mu$.

(3) If $\mu$ is a fuzzy semidense set on $X$, then $f(\mu)$ is a fuzzy semidense set on $Y$.

Proof. (1) implies (2): Let $\nu$ be a fuzzy semiclosed set on $Y$ such that $f^{-1}(\nu) \neq 1_{X}$. Then $\nu^{c}$ is a fuzzy semiopen set on $Y$ and $f^{-1}\left(\nu^{c}\right)=\left(f^{-1}(\nu)\right)^{c} \neq 0_{X}$. Since $f$ is somewhat fuzzy irresolute continuous, there exists a fuzzy semiopen set $\lambda \neq 0_{X}$ on $X$ such that $\lambda \leq f^{-1}\left(\nu^{c}\right)$. Let $\mu=\lambda^{c}$. Then $\mu \neq 1_{X}$ is fuzzy semiclosed such that $f^{-1}(\nu)=1-f^{-1}\left(\nu^{c}\right) \leq 1-\lambda=\lambda^{c}=\mu$.

(2) implies (3): Let $\mu$ be a fuzzy semidense set on $X$ and suppose that $f(\mu)$ is not fuzzy semidense on $Y$. Then there exists a fuzzy semiclosed set $\nu$ on $Y$ such that $f(\mu)<\nu<1$. Since $\nu<1$ and $f^{-1}(\nu) \neq 1_{X}$, there exists a fuzzy semiclosed set $\delta \neq 1_{X}$ such that $\mu \leq f^{-1}(f(\mu))<f^{-1}(\nu) \leq \delta$. This contradicts to the assumption that $\mu$ is a fuzzy semidense set on $X$. Hence $f(\mu)$ is a fuzzy semidense set on $Y$.

(3) implies (1): Let $\nu \neq 0_{Y}$ be a fuzzy semiopen set on $Y$ and let $f^{-1}(\nu) \neq 0_{X}$. Suppose that there exists no fuzzy semiopen $\mu \neq 0_{X}$ on $X$ such that $\mu \leq f^{-1}(\nu)$. Then $\left(f^{-1}(\nu)\right)^{c}$ is a fuzzy set on $X$ such that there is no fuzzy semiclosed set $\delta$ on $X$ with $\left(f^{-1}(\nu)\right)^{c}<\delta<1$. In fact, if there exists a fuzzy semiopen set $\delta^{c}$ such that $\delta^{c} \leq f^{-1}(\nu)$, then it is a contradiction. So $\left(f^{-1}(\nu)\right)^{c}$ is a fuzzy semidense set on $X$. Hence $f\left(\left(f^{-1}(\nu)\right)^{c}\right)$ is a fuzzy semidense set on $Y$. But $f\left(\left(f^{-1}(\nu)\right)^{c}\right)=f\left(f^{-1}\left(\nu^{c}\right)\right) \neq \nu^{c}<1$. This is a contradiction to the fact that $f\left(\left(f^{-1}(\nu)\right)^{c}\right)$ is fuzzy semidense on $Y$. Hence there exists a semiopen set $\mu \neq 0_{X}$ on $X$ such that $\mu \leq f^{-1}(\nu)$. Consequently, $f$ is somewhat fuzzy irresolute continuous.

Theorem 3.6. Let $X_{1}$ be product related to $X_{2}$ and let $Y_{1}$ be product related to $Y_{2}$. Then the product $f_{1} \times f_{2}$ : $X_{1} \times X_{2} \rightarrow Y_{1} \times Y_{2}$ of somewhat fuzzy irresolute continuous mappings $f_{1}: X_{1} \rightarrow Y_{1}$ and $f_{2}: X_{2} \rightarrow Y_{2}$ is also somewhat fuzzy irresolute continuous.

Proof. Let $\lambda=\bigvee_{i, j}\left(\mu_{i} \times \nu_{j}\right)$ be a fuzzy semiopen set on $Y_{1} \times Y_{2}$ where $\mu_{i} \neq 0_{Y_{1}}$ and $\nu_{j} \neq 0_{Y_{2}}$ are fuzzy semiopen sets on $Y_{1}$ and $Y_{2}$ respectively. Then $\left(f_{1} \times f_{2}\right)^{-1}(\lambda)=$ $\bigvee_{i, j}\left(f_{1}^{-1}\left(\mu_{i}\right) \times f_{2}^{-1}\left(\nu_{j}\right)\right)$. Since $f_{1}$ is somewhat fuzzy irresolute continuous, there exists a fuzzy semiopen set $\delta_{i} \neq 0_{X_{1}}$ such that $\delta_{i} \leq f_{1}^{-1}\left(\mu_{i}\right) \neq 0_{X_{1}}$. And, since $f_{2}$ is somewhat fuzzy irresolute continuous, there exists a fuzzy semiopen set $\eta_{j} \neq 0_{X_{2}}$ such that $\eta_{j} \leq f_{2}^{-1}\left(\nu_{j}\right) \neq 0_{X_{2}}$. Now $\delta_{i} \times \eta_{j} \leq f_{1}^{-1}\left(\mu_{i}\right) \times f_{2}^{-1}\left(\nu_{j}\right)=\left(f_{1} \times f_{2}\right)^{-1}\left(\mu_{i} \times\right.$ $\left.\nu_{j}\right)$ and $\delta_{i} \times \eta_{j} \neq 0_{X_{1} \times X_{2}}$ is a fuzzy semiopen set on $X_{1} \times X_{2}$. Hence $\bigvee_{i, j}\left(\delta_{i} \times \eta_{j}\right) \neq 0_{X_{1} \times X_{2}}$ is a fuzzy semiopen set on $X_{1} \times X_{2}$ such that $\bigvee_{i, j}\left(\delta_{i} \times \eta_{j}\right) \leq$ $\bigvee_{i, j}\left(f_{1}^{-1}\left(\mu_{i}\right) \times f_{2}^{-1}\left(\nu_{j}\right)\right)=\left(f_{1} \times f_{2}\right)^{-1}\left(\bigvee_{i, j}\left(\mu_{i} \times \nu_{j}\right)\right)=$ $\left(f_{1} \times f_{2}\right)^{-1}(\lambda) \neq 0_{X_{1} \times X_{2}}$. Therefore, $f_{1} \times f_{2}$ is somewhat fuzzy irresolute continuous.

Theorem 3.7. Let $f: X \rightarrow Y$ be a mapping. If the graph $g: X \rightarrow X \times Y$ of $f$ is a somewhat fuzzy irresolute continuous mapping, then $f$ is also somewhat fuzzy irresolute continuous.

Proof. Let $\nu$ be a fuzzy semiopen set on $Y$. Then $f^{-1}(\nu)=1 \wedge f^{-1}(\nu)=g^{-1}(1 \times \nu)$. Since $g$ is somewhat fuzzy irresolute continuous and $1 \times \nu$ is a fuzzy semiopen set on $X \times Y$, there exists a fuzzy semiopen set $\mu \neq 0_{X}$ on $X$ such that $\mu \leq g^{-1}(1 \times \nu)=f^{-1}(\nu) \neq 0_{X}$. Therefore, $f$ is somewhat fuzzy irresolute continuous.

Definition 3.8. A mapping $f: X \rightarrow Y$ is called somewhat fuzzy irresolute semiopen if there exists a fuzzy semiopen set $\nu \neq 0_{Y}$ on $Y$ such that $\nu \leq f(\mu) \neq 0_{Y}$ for any fuzzy semiopen set $\mu \neq 0_{X}$ on $X$.

It is clear that every fuzzy irresolute semiopen mapping is a somewhat fuzzy irresolute semiopen mapping. And every somewhat fuzzy irresolute semiopen mapping is a 
fuzzy semiopen mapping. Also, every fuzzy semiopen mapping is a somewhat fuzzy semiopen mapping from the above definitions. But the converses are not true in general as the following examples show.

Example 3.9. Let $\mu_{1}, \mu_{2}$ and $\mu_{3}$ be fuzzy sets on $X=$ $\{a, b, c\}$ and let $\nu_{1}, \nu_{2}$ and $\nu_{3}$ be fuzzy sets on $Y=$ $\{x, y, z\}$ with

$$
\begin{aligned}
& \mu_{1}(a)=0.1, \mu_{1}(b)=0.1, \mu_{1}(c)=0.1, \\
& \mu_{2}(a)=0.2, \mu_{2}(b)=0.2, \mu_{2}(c)=0.2, \\
& \mu_{3}(a)=0.5, \mu_{3}(b)=0.5, \mu_{3}(c)=0.5 \text { and } \\
& \nu_{1}(x)=0.0, \nu_{1}(y)=0.1, \nu_{1}(z)=0.0, \\
& \nu_{2}(x)=0.0, \nu_{2}(y)=0.2, \nu_{2}(z)=0.0, \\
& \nu_{3}(x)=0.0, \nu_{3}(y)=0.5, \nu_{3}(z)=0.0 .
\end{aligned}
$$

Let $\tau=\left\{0_{X}, \mu_{1}, \mu_{2}, 1_{X}\right\}$ be fuzzy topologies on $X$ and let $\tau^{*}=\left\{0_{Y}, \nu_{1}, \nu_{1}^{c}, 1_{Y}\right\}$ be fuzzy topologies on $Y$. Consider the mapping $f:(X, \tau) \rightarrow\left(Y, \tau^{*}\right)$ defined by $f(a)=y$, $f(b)=y$ and $f(c)=y$. Since $f\left(\mu_{1}\right)=\nu_{1}, \nu_{1} \leq f\left(\mu_{2}\right)=$ $\nu_{2}$ and $\nu_{1} \leq f\left(\mu_{3}\right)=\nu_{3}, f$ is somewhat fuzzy irresolute semiopen. But $f\left(\mu_{2}\right)=\nu_{2}$ and $f\left(\mu_{3}\right)=\nu_{3}$ are not fuzzy semiopen sets. Hence $f$ is not a fuzzy irresolute semiopen mapping.

Example 3.10. Let $\mu_{1}, \mu_{2}$ and $\mu_{3}$ be fuzzy sets on $I=$ $[0,1]$ with

$$
\begin{aligned}
& \mu_{1}(x)=0.4,0 \leq x \leq 1, \\
& \mu_{2}(x)=0.5,0 \leq x \leq 1 \text { and } \\
& \mu_{3}(x)=0.6,0 \leq x \leq 1
\end{aligned}
$$

Let $\tau=\left\{0_{I}, \mu_{1}, 1_{I}\right\}$ and $\tau^{*}=\left\{0_{I}, \mu_{3}, 1_{I}\right\}$ be fuzzy topologies on $I$. Consider the mapping $f:(I, \tau) \rightarrow$ $\left(I, \tau^{*}\right)$ defined by $f(x)=1-x, 0 \leq x \leq 1$. Since $f\left(\mu_{1}\right)=\mu_{3}$ is a fuzzy semiopen set on $\left(I, \tau^{*}\right), f$ : $(I, \tau) \rightarrow\left(I, \tau^{*}\right)$ is fuzzy semiopen. But $\mu_{2}$ is a fuzzy semiopen set on $(I, \tau)$ and $f\left(\mu_{2}\right)=\mu_{2}$ is not a fuzzy semiopen set on $(I, \tau *)$. Also, there is no non-zero fuzzy semiopen set smaller than $f\left(\mu_{2}\right)=\mu_{2} \neq 0$. Hence $f$ is not a somewhat fuzzy irresolute semiopen mapping.

Example 3.11. Let $\mu_{1}, \mu_{2}$ and $\mu_{3}$ be fuzzy sets on $X=$ $\{a, b, c\}$ with

$$
\begin{aligned}
& \mu_{1}(a)=0.1, \mu_{1}(b)=0.1, \mu_{1}(c)=0.1, \\
& \mu_{2}(a)=0.3, \mu_{2}(b)=0.3, \mu_{2}(c)=0.3 \text { and } \\
& \mu_{3}(a)=0.8, \mu_{3}(b)=0.8, \mu_{3}(c)=0.8 .
\end{aligned}
$$

Let $\tau=\left\{0_{X}, \mu_{3}, 1_{X}\right\}$ and $\tau^{*}=\left\{0_{X}, \mu_{1}, \mu_{2}, 1_{X}\right\}$ be fuzzy topologies on $X$. Consider the identity mapping
$i_{X}:(X, \tau) \rightarrow\left(X, \tau^{*}\right)$. Then we have $\mu_{2}^{c} \leq i_{X}\left(\mu_{3}\right)=\mu_{3}$. Since $\mu_{2}^{c}$ is a fuzzy semiopen set on $(X, \tau), i_{X}$ is somewhat fuzzy semiopen. But $i_{X}\left(\mu_{3}\right)=\mu_{3}$ is not a fuzzy semiopen set on $\left(X, \tau^{*}\right)$. Hence $i_{X}$ is not a fuzzy semiopen mapping.

Theorem 3.12. Let $f: X \rightarrow Y$ be a bijection. Then the following are equivalent:

(1) $f$ is somewhat fuzzy irresolute semiopen.

(2) If $\mu$ is a fuzzy semiclosed set on $X$ such that $f(\mu) \neq$ $1_{Y}$, then there exists a fuzzy semiclosed set $\nu \neq 1_{Y}$ on $Y$ such that $f(\mu)<\nu$.

Proof. (1) implies (2): Let $\mu$ be a fuzzy semiclosed set on $X$ such that $f(\mu) \neq 1_{Y}$. Since $f$ is bijective and $\mu^{c}$ is a fuzzy semiopen set on $X, f\left(\mu^{c}\right)=(f(\mu))^{c} \neq 0_{Y}$. And, since $f$ is somewhat fuzzy irresolute semiopen, there exists a semiopen set $\delta \neq 0_{Y}$ on $Y$ such that $\delta<f\left(\mu^{c}\right)=$ $(f(\mu))^{c}$. Consequently, $f(\mu)<\delta^{c}=\nu \neq 1_{Y}$ and $\nu$ is a fuzzy semiclosed set on $Y$.

(2) implies (1): Let $\mu$ be a fuzzy semiopen set on $X$ such that $f(\mu) \neq 0_{Y}$. Then $\mu^{c}$ is a fuzzy semiclosed set on $X$ and $f\left(\mu^{c}\right) \neq 1_{Y}$. Hence there exists a fuzzy semiclosed set $\nu \neq 1_{Y}$ on $Y$ such that $f\left(\mu^{c}\right)<\nu$. Since $f$ is bijective, $f\left(\mu^{c}\right)=(f(\mu))^{c}<\nu$. Thus $\nu^{c}<f(\mu)$ and $\nu^{c} \neq 0_{X}$ is a fuzzy semiopen set on $Y$. Therefore, $f$ is somewhat fuzzy irresolute semiopen.

Theorem 3.13. Let $f: X \rightarrow Y$ be a surjection. Then the following are equivalent:

(1) $f$ is somewhat fuzzy irresolute semiopen.

(2) If $\nu$ is a fuzzy semidense set on $Y$, then $f^{-1}(\nu)$ is a fuzzy semidense set on $X$.

Proof. (1) implies (2): Let $\nu$ be a fuzzy semidense set on $Y$. Suppose $f^{-1}(\nu)$ is not fuzzy semidense on $X$. Then there exists a fuzzy semiclosed set $\mu$ on $X$ such that $f^{-1}(\nu)<\mu<1$. Since $f$ is somewhat fuzzy irresolute semiopen and $\mu^{c}$ is a fuzzy semiopen set on $X$, there exists a fuzzy semiopen set $\delta \neq 0_{Y}$ on $Y$ such that $\delta \leq f\left(\operatorname{Int} \mu^{c}\right) \leq f\left(\mu^{c}\right)$. Since $f$ is surjective, $\delta \leq f\left(\mu^{c}\right)<$ $f\left(f^{-1}\left(\nu^{c}\right)\right)=\nu^{c}$. Thus there exists a semiclosed set $\delta^{c}$ on $Y$ such that $\nu<\delta^{c}<1$. This is a contradiction. Hence $f^{-1}(\nu)$ is fuzzy semidense on $X$.

(2) implies (1): Let $\mu$ be a fuzzy open set on $X$ and $f(\mu) \neq 0_{Y}$. Suppose there exists no fuzzy semiopen $\nu \neq$ $0_{Y}$ on $Y$ such that $\nu \leq f(\mu)$. Then $(f(\mu))^{c}$ is a fuzzy set on $Y$ such that there exists no fuzzy semiclosed set $\delta$ on $Y$ with $(f(\mu))^{c}<\delta<1$. This means that $(f(\mu))^{c}$ is fuzzy semidense on $Y$. Thus $f^{-1}\left((f(\mu))^{c}\right)$ is fuzzy semidense on $X$. But $f^{-1}\left((f(\mu))^{c}\right)=\left(f^{-1}(f(\mu))\right)^{c} \leq \mu^{c}<1$. This is a contradiction to the fact that $f^{-1}\left((f(\nu))^{c}\right.$ is fuzzy 
semidense on $X$. Hence there exists a semiopen set $\nu \neq 0_{Y}$ on $Y$ such that $\nu \leq f(\mu)$. Therefore, $f$ is somewhat fuzzy irresolute semiopen.

\section{References}

[1] K. K. Azad, "On fuzzy semicontinuity, fuzzy almost continuity and fuzzy weakly continuity," J. Math. Anal. Appl., vol. 82, pp. 14-32, 1981.

[2] Karl R. Gentry and Hughes B. Hoyle, III, "Somewhat continuous functions," Czech. Math. J., vol. 21, pp. 512, 1971.

[3] M. N. Mukherjee and S. P. Sinha, "Irresolute and almost open functions between fuzzy topological spaces," Fuzzy Sets and Systems, vol. 29, pp. 381-388, 1989.

[4] G. Thangaraj and G. Balasubramanian, "On somewhat fuzzy $\alpha$-continuous functions," J. Fuzzy Math., vol. 16, pp. 641-651, 2008.

[5] _ , "On Somewhat fuzzy continuous functions," J. Fuzzy Math., vol. 11, pp. 725-736, 2003.
[6] _ , "On Somewhat fuzzy semicontinuous functions," Kybernetika , vol. 137, pp. 165-170, 2001.

\section{Young Bin Im}

Faculty of General Education, Dongguk University, Seoul 100-715, Korea

Research Area: Fuzzy topology, Fuzzy matrix

E-mail : philpen@dongguk.edu

\section{Joo Sung Lee}

Department of Mathematics, Dongguk University, Seoul 100-715, Korea

Research Area: Fuzzy topology, Topological dynamics

E-mail : jsl@dongguk.edu

\section{Yung Duk Cho}

Faculty of General Education, Dongguk University, Seoul 100-715, Korea

Research Area: Fuzzy topology, Fuzzy algebra

E-mail : joyd@dongguk.edu 